\title{
Parker Solar Probe's Measurements of the 29 November 2020 Solar Energetic Particle Event
}

C.M.S. Cohen ${ }^{a *}$, E.R. Christian ${ }^{b}$, A.C. Cummings ${ }^{a}$, A.J. Davis ${ }^{a}$, M.I. Desaic, G.A. de Nolfo ${ }^{b}$, J. Giacalone ${ }^{d}$, M.E. Hill ${ }^{e}$, C.J. Joyce ${ }^{f}$, A.W. Labrador ${ }^{a}$, R.A. Leske ${ }^{a}$, W.H. Matthaeus ${ }^{g}$, D.J. McComas ${ }^{f}$, R.L. McNutt Jr. ${ }^{e}$, R.A. Mewaldt ${ }^{a}$, D.G. Mitchell ${ }^{e}$, J.G. Mitchell ${ }^{b, h}$, J.S. Rankin ${ }^{f}$, E.C. Roelof ${ }^{e}$, N.A. Schwadron', E.C. Stone ${ }^{a}$, J.R. Szalay ${ }^{f}$, M.E. Wiedenbeck', A. Vourlidas ${ }^{e}$, S.D. Bale ${ }^{k, l}$, M. Pulupa', and R.J. MacDowall

${ }^{a}$ California Institute of Technology, Pasadena, CA USA

${ }^{b} N A S A /$ Goddard Space Flight Center, Greenbelt, MD USA

${ }^{c}$ University of Texas at San Antonio, San Antonio, TX USA

${ }^{d}$ University of Arizona, Tucson, AZ USA

e Johns Hopkins University Applied Physics Laboratory, Laurel, MD USA

${ }^{f}$ Department of Astrophysical Sciences, Princeton University, Princeton, NJ USA

${ }^{g}$ University of Delaware, Newark, DE USA

${ }^{h}$ Department of Physics, George Washington University, Washington, DC USA

${ }^{i}$ University of New Hampshire, Durham, NH USA

jJet Propulsion Laboratory, California Institute of Technology, Pasadena, CA USA

${ }^{k}$ Physics Department, University of California at Berkeley, Berkeley, CA USA

${ }^{l}$ Space Sciences Laboratory, University of California at Berkeley, Berkeley, CA USA

E-mail: cohenesrl.caltech.edu 
On November 29, 2020 active region 12790 was located just beyond the east limb of the Sun as viewed by Earth. It erupted at 12:34UT with an M4.4 flare (as measured by GOES) and launched a coronal mass ejection (CME) traveling $\sim 1700 \mathrm{~km} / \mathrm{s}$. Not surprisingly, this fast CME drove a shock that accelerated particles up to tens of $\mathrm{MeV} /$ nuc. More unusual was that these solar energetic particles (SEPs) quickly filled the inner heliosphere and the event was observed by spacecraft distributed around the Sun, including Parker Solar Probe (PSP), STEREO-A, Solar Orbiter, and those near Earth such as ACE and SOHO. This was the first mid-sized SEP event detected by the Integrated Science Investigation of the Sun (IS@IS) suite on PSP and its first opportunity to make measurements of heavy ion spectra up to tens of $\mathrm{MeV} /$ nuc. Here we present an overview of event characteristics as determined by IS@IS, including $\mathrm{H}, \mathrm{He}, \mathrm{O}$, and Fe spectra, composition as a function of energy, and temporal variations of the energetic particle intensities throughout the event.

37th International Cosmic Ray Conference (ICRC 2021)

July 12th-23rd, 2021

Online - Berlin, Germany 


\section{Introduction}

The Parker Solar Probe (PSP) mission [1] was launched on 12 August 2018 with four suites of instruments designed to investigate the origin of the solar wind, its structures and dynamics, and how energetic particles are accelerated and transported. A key aspect of the mission is obtaining perihelion distances $<10 \mathrm{R}_{\mathrm{S}}$ through a series of Venus flybys over the course of the mission's 24 orbits. The solar wind plasma is measured by instruments comprising the Solar Wind Electron Alpha Proton (SWEAP) suite [2]; the magnetic fields and radio emissions are measured by the Electromagnetic Fields Investigation (FIELDS) suite [3]; and the energetic particles are measured by sensors in the Integrated Science Investigation of the Sun (IS@IS) suite [4].

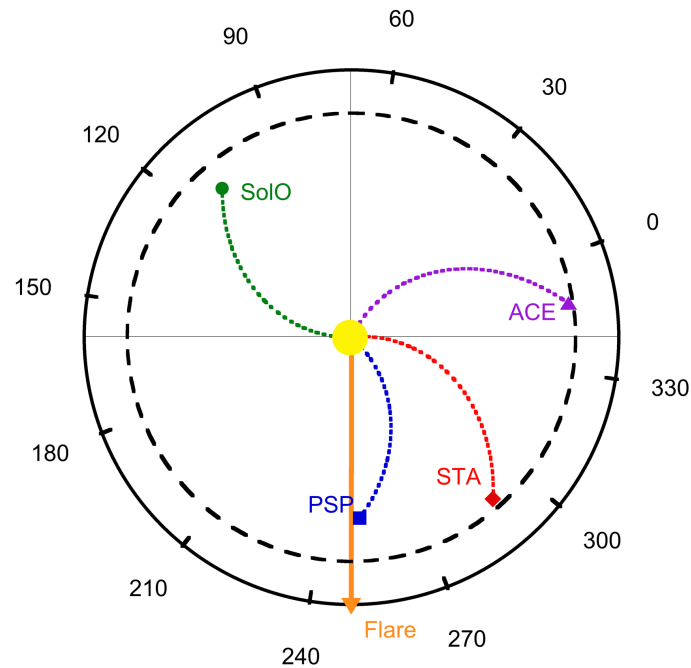

Fig 1. Position of various spacecraft on 29 November 2020 12:34UT. The longitude of the flare is indicated by the orange arrow. The dashed circle marks $1 \mathrm{AU}$ and the outer numbers mark Carrington longitude.

During the first several orbits, solar activity was fairly low and energetic particle observations were limited to primarily protons and helium measured during stream interaction regions and small solar energetic particle (SEP) events (see, e.g., [5]), often at energies below $\sim 10 \mathrm{MeV} /$ nuc. During its $7^{\text {th }}$ orbit, IS@IS observed its first mid-sized SEP event, with heavy ions measured at energies of tens of $\mathrm{MeV} /$ nuc and protons at $>40 \mathrm{MeV}$. Although PSP was not particularly close to the Sun at this time (being at $0.8 \mathrm{AU}$ ), the event provided the opportunity to calibrate the heavy ion response of the IS@IS sensors. Additionally, the event was sufficiently large that it was observed by multiple spacecraft distributed around the Sun (Figure 1), allowing studies of SEP characteristics as a function of longitude to be made (see, e.g., [6, 7, 8]). The IS๑IS observations of this event are presented in detailed in [9]; here we provide a brief overview of the key characteristics of this event.

\section{Observations}

\subsection{Solar}

Although active region 12790 was just behind the east limb (E99S23) as viewed from Earth, the GOES-16 x-ray instrument registered an M4.4 class flare (Figure 2b) when it erupted on 29 November 2020. The flare peaked at 13:11UT and was associated with a coronal mass ejection (CME), observed by SOHO/LASCO and STEREO-A/COR2. Sitting $58^{\circ}$ east of the Sun-Earth line, STEREO-A had a good view of the active region and its erupting filament (Figure 2a). LASCO's nearly orthogonal view of the CME provided the best measure of the speed as projection effects were minimal. The speed of the CME front was calculated to be $\sim 1700 \mathrm{~km} / \mathrm{s}$ and decelerated to $1500 \mathrm{~km} / \mathrm{s}$ when it passed out of LASCO's field of view. The Radio Frequency Spectrometer (RFS, [10]) of FIELDS observed several type III bursts and a type II burst associated with the solar activity (Figure 2c,d). 

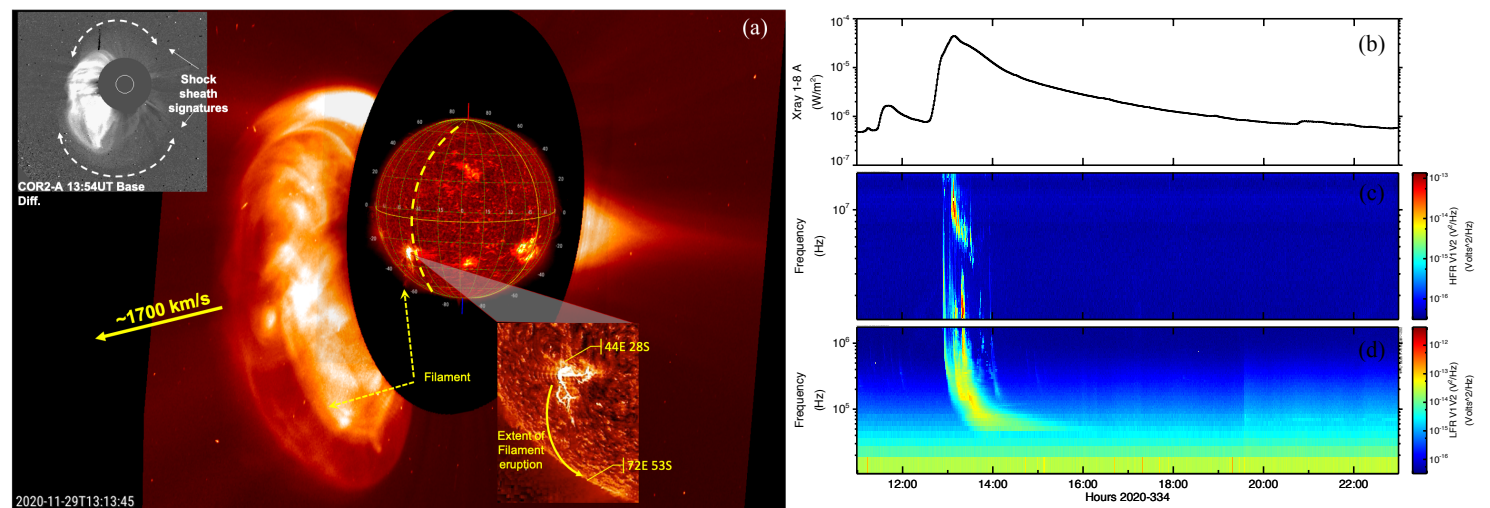

Fig. 2. (a) Overview of the erupting filament and corresponding CME from the STEREO-A point of view. The inner solar image is a composite of SDO/AIA and STEREO-A/EUVI 304 $\AA$, with the east limb as viewed from Earth indicated by the dashed yellow arc. The bottom-right insert shows a close-up of the active region with the yellow arrow indicating the extent of the filament eruption. The upper-left insert shows the STEREO-A/COR2 image of the CME. (b) GOES-16 soft x-ray time profile. (c) The PSP high frequency radio observations; (d) the low frequency radio data.

\subsection{In-situ}

The IS@IS suite is comprised of two instruments, Energetic Particle Instrument-Low (EPILo, [11]) and Energetic Particle Instrument-High (EPI-Hi, [12]) which together measure energetic ions $\mathrm{H}-\mathrm{Fe}$ at energies of $\sim 20 \mathrm{keV}$ to $>100 \mathrm{MeV} /$ nuc and energetic electrons from $\sim 15 \mathrm{keV}$ to $\sim 6$ $\mathrm{MeV}$. EPI-Lo utilizes the time-of-flight versus energy technique in 80 separate apertures to obtain a $2 \pi$-sr field of view. EPI-Hi has five apertures (which all use the $\mathrm{dE} / \mathrm{dx}$ versus residual energy

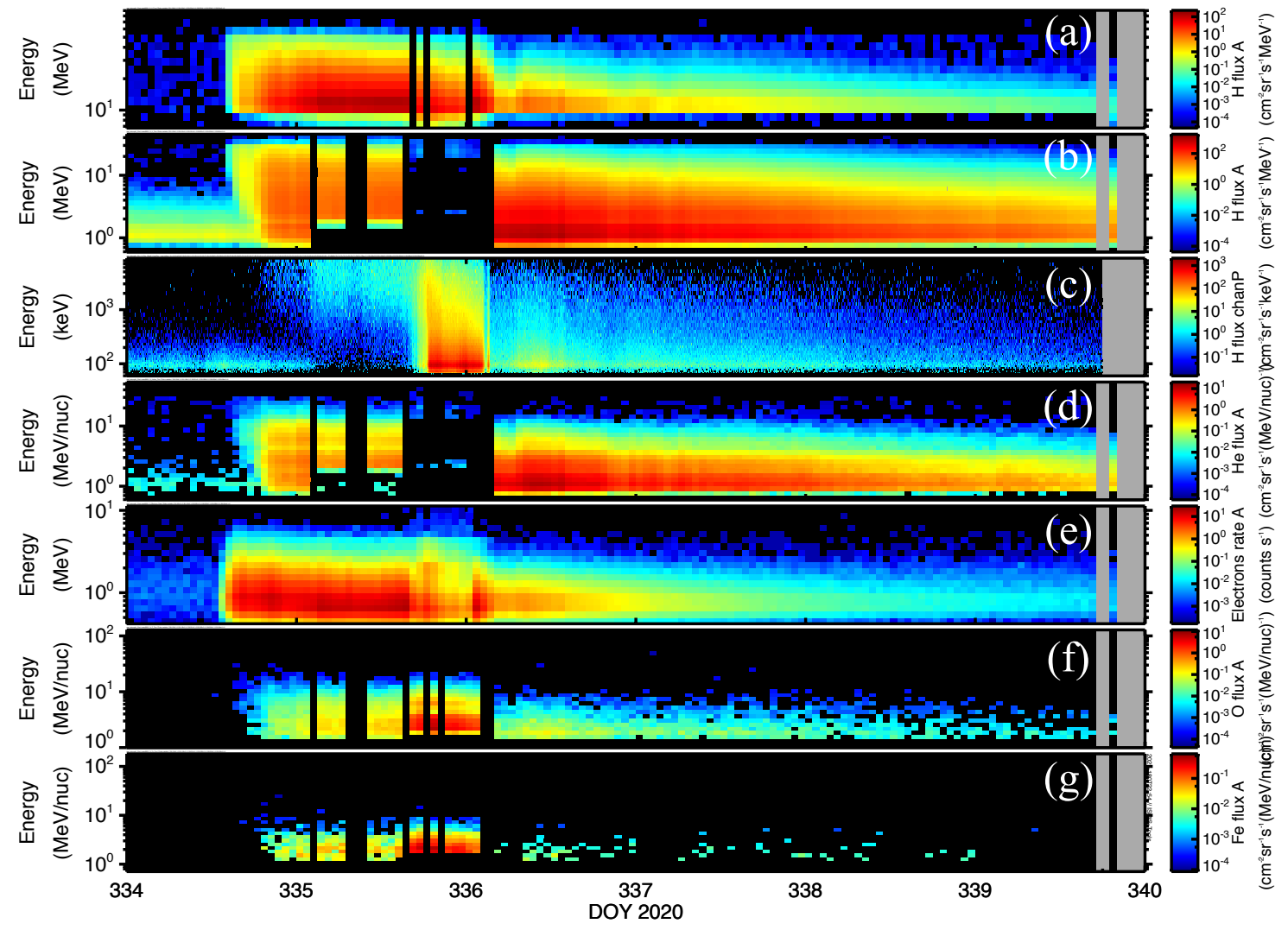

Fig. 3. Spectrograms for H from HETA (a), LETA (b), EPI-Lo (c); He from LETA (d); electrons from HETA (e); O and Fe from LETA (f, g, respectively). Black bands correspond to data gaps due to instruments changing dynamic threshold modes. See text regarding panels (b), (d). 

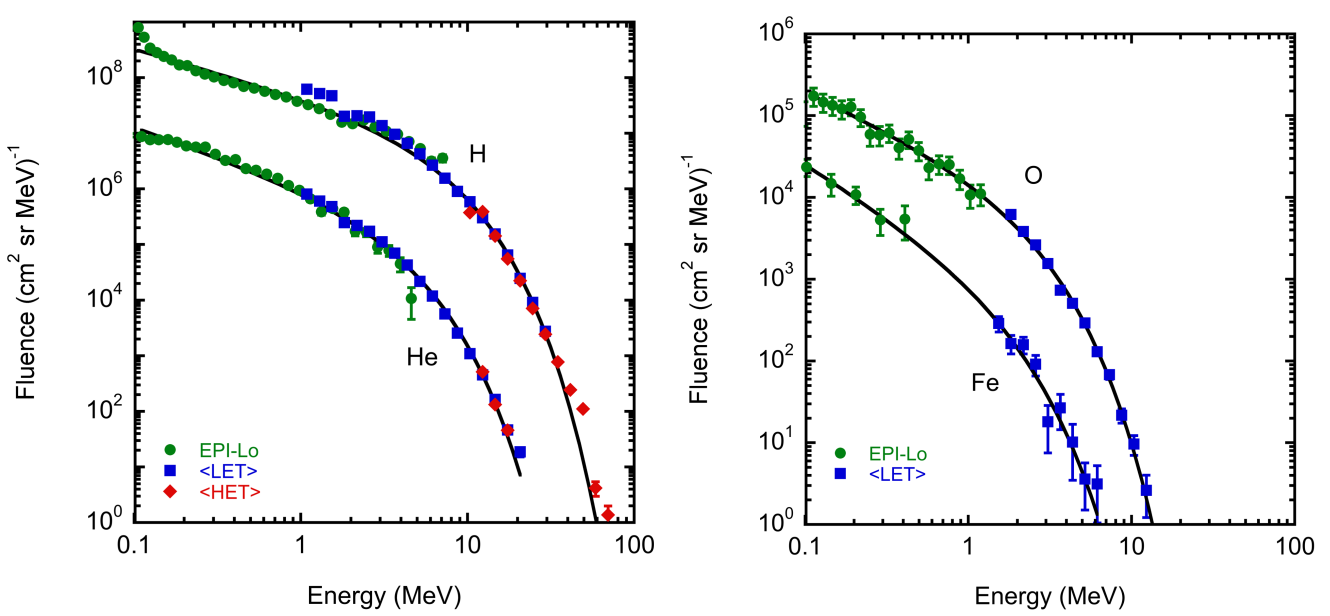

Fig 4. Spectra integrated over the decay period for $\mathrm{H}, \mathrm{He}, \mathrm{O}$, and $\mathrm{Fe}$. Note $\mathrm{O}$ and $\mathrm{Fe}$ intensities were not high enough to be measured by HET.

technique): three Low Energy Telescopes (LETA, LETB, and LETC) measure ions starting at $~ 1$ $\mathrm{MeV} /$ nuc and two High Energy Telescopes (HETA and HETB) which measure ions $>10 \mathrm{MeV} /$ nuc. LETA and LETB view in opposite directions as do HETA and HETB (LETC looks orthogonally to LETA); LETA and HETA are mounted with their axes $45^{\circ}$ and $20^{\circ}$, respectively, relative to the spacecraft's Z-axis (nominally pointed towards the Sun).

An overview of the SEP event is shown in Figure 3, with spectrogram data from HETA, LETA, and EPI-Lo (integrated over all apertures) for e-, $\mathrm{H}, \mathrm{He}, \mathrm{O}$ and Fe. The odd features seen in LETA $\mathrm{H}$ and $\mathrm{He}$ (panels $\mathrm{b}$ and $\mathrm{d}$ ) are the result of EPI-Hi going into different dynamic threshold modes. These modes are triggered when the count rate of ions entering EPI-Hi are high enough to cause significant deadtime in the instrument. When this occurs, the energy thresholds of selected detectors are raised to reduce the instrument's response to e-, $\mathrm{H}$, and $\mathrm{He}$, thus improving the instrument livetime and prioritizing the $Z>2$ ions. The November event was the first event large enough to trigger the dynamic threshold system and it was found that due to a software issue the LETs did not perform as expected in dynamic threshold level 2. While this has since been corrected, it resulted in the substantial loss of e-, $\mathrm{H}$, and He statistics from LETA and LETB during the period 30 November (day 335) 15:00UT - 1 December (day 336) 03:00UT. HETA and HETB

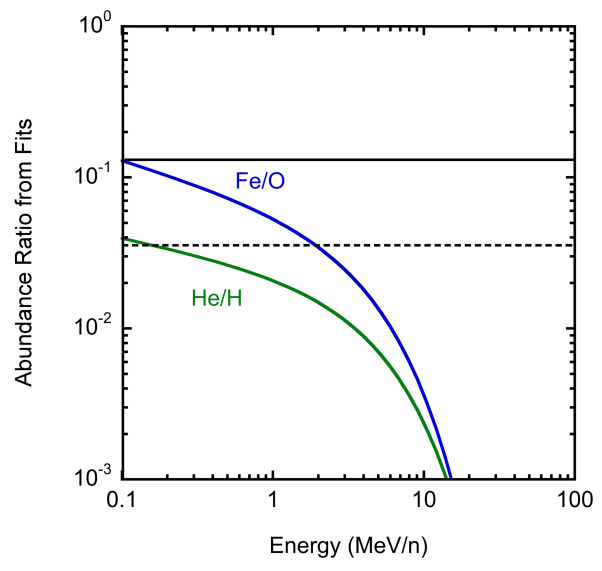

Fig. 5. $\mathrm{He} / \mathrm{H}$ and $\mathrm{Fe} / \mathrm{O}$ abundance ratios vs energy (from fits in Figure 4). Solid and dashed horizontal lines mark abundances of $\mathrm{Fe} / \mathrm{O}$ and $\mathrm{He} / \mathrm{H}$, respectively, from [16]. also exercised dynamic thresholds but only at level 1, which resulted in decreased geometry factors which have been incorporated into the data analysis presented below.

Due to these instrumental mode changes, we focus on the decay portion of the event (December 1 (day 336), 04:00UT - December 4 (day 339), 17:00UT) for the spectra and composition analysis. During this period, the particle intensities measured by LETA and LETB (and similarly for HETA and HETB) are similar suggesting an isotropic particle distribution; thus, we use the average of the $\mathrm{A}$ and $\mathrm{B}$ apertures to create $<$ LET $>$ and $<$ HET $>$ intensities. By integrating these, along with the EPI-Lo omni-directional intensities, over 


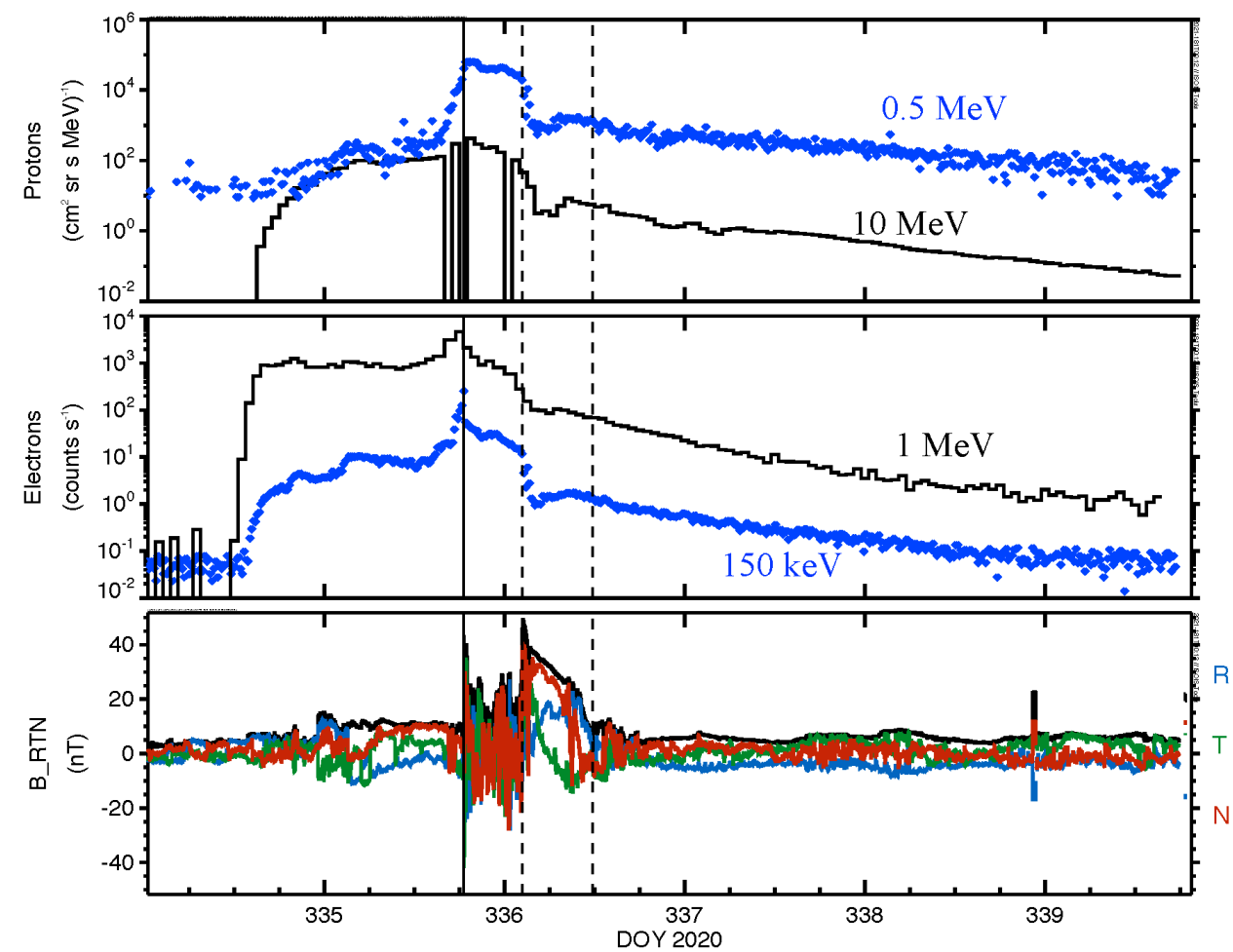

Fig. 6. Proton intensities (top) and electron count rates (middle) from EPI-Lo (blue) and EPI-Hi (black). Bottom panel shows components of the magnetic field. Solid line marks the interplanetary shock and dashed lines bound the magnetic cloud period.

the decay phase, we obtain the fluence spectra for $\mathrm{H}, \mathrm{He}, \mathrm{O}$, and Fe shown in Figure 4 (n. b., the $\mathrm{O}$ and $\mathrm{Fe}$ intensities were not sufficiently high enough at $>10 \mathrm{MeV} /$ nuc to be measured by HET). The spectra are all well fit with the Ellison \& Ramaty [13] spectral form (represented as a power law multiplied by an exponential) with spectral breaks that decrease in energy with increasing particle mass. This effect results in energy-dependent composition ratios as illustrated in Figure 5 for $\mathrm{He} / \mathrm{H}$ and $\mathrm{Fe} / \mathrm{O}$ as is commonly seen in shock-accelerated SEP events (see, e.g., $[14,15]$ ). At $100 \mathrm{keV} / \mathrm{nuc}$ the abundances agree well with the average ratios for shock-accelerated SEP events as reported by [16].

The overall time profile of the event (Figure 6) shows a number of features in the protons and electrons associated with magnetic structures. The pattern of an initial increase followed by a strong peak occurring at the time of the shock passage (marked by the solid line at November 30 (day 335), 18:35UT) is typical for SEP events generated by a CME-driven shock from an active region located near spacecraft's central meridian (i.e., longitude of the Sun-spacecraft line), as was the case for this event. The nearly flat intensities of the $0.5 \mathrm{MeV}$ protons followed by the strong increase as the shock approaches is consistent with efficient confinement of the particles to the shock region (see [8] for more details). Somewhat surprising is the peak observed in both electron count rates with the passage of the shock. As interplanetary shocks are not often found to accelerate electrons [17], it is more likely that this is a result of the electrons being trapped in the vicinity of the shock.

Figure 7 zooms in on the sheath and magnetic cloud portion of the event, using 1-minute engineering rates from HET (the standard science data are only 1-hour resolution). The drop in proton and electron intensities at all energies coinciding with the onset of the magnetic cloud (line a) is fairly common (see, e.g., [18]). Shortly thereafter there is a $\sim 30$ minute period (delineated 


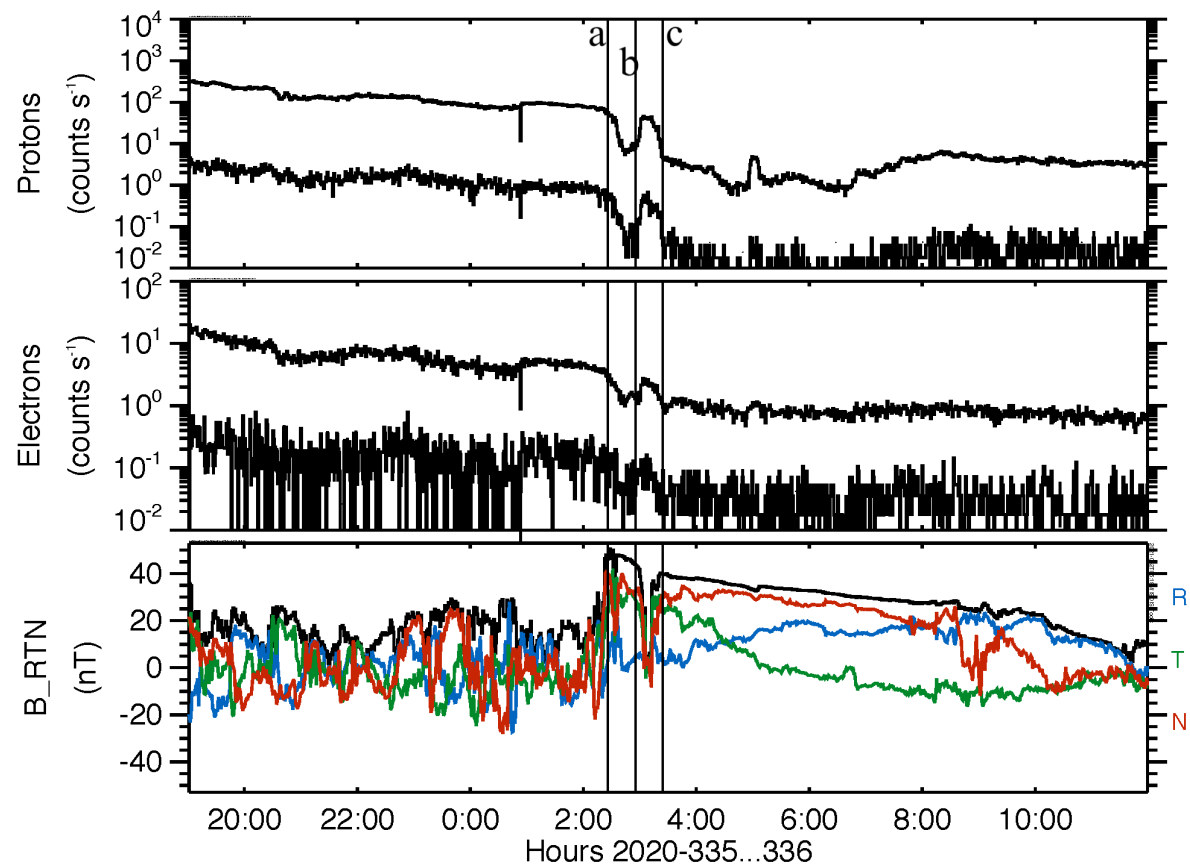

Fig. 7. The sheath and cloud portion of the event as measured in 1-minute rates of protons stopping in the third and fifth detector of HET (upper and lower traces, respectively, top panel) and electrons stopping in the third and fourth HET detectors (upper and lower traces, respectively, second panel). The bottom panel shows the magnitude and components of the magnetic field. Vertical lines indicate features seen in the particle rates that mark changes in the magnetic field discussed in the text.

by lines $\mathrm{b}$ and $\mathrm{c}$ ) where all the particle intensities recover to approximately their pre-magnetic cloud values. This period is also marked by a drop in the magnetic field strength, again to values similar to that outside the magnetic cloud. These signatures suggest that PSP briefly exited the magnetic cloud and then returned.

\section{Discussion and Summary}

The 29 November 2020 SEP event was the first mid-sized SEP event observed by IS@IS, allowing the EPI-Lo and EPI-Hi response to heavy ions to be partially calibrated (as the heavy ions did not extend to the HET energies, the heavy ion calibration for HET remains incomplete). The combined IS@IS spectra for $\mathrm{H}, \mathrm{He}, \mathrm{O}$ and Fe obtained during the decay of the event are found to be well described by the Ellison \& Ramaty form, with spectral breaks that decrease in energy with increasing mass. This leads to energy-dependent abundance ratios for $\mathrm{He} / \mathrm{H}$ and $\mathrm{Fe} / \mathrm{O}$ with values at $100 \mathrm{keV} /$ nuc typical of shock-accelerated SEP events. The overall time profile of the particle intensities is as expected given the relative locations of PSP and the active region with the particle intensities peaking at the passage of the interplanetary shock indicating significant confinement of particles to the shock region. The event decay is interrupted by a brief excursion of the spacecraft out of the magnetic cloud as indicated by a period of increased particle intensities and decreased magnetic field.

A number of interesting details regarding this event are being studied. These include the period shortly before and after the shock and associated SEP intensity variations [19] and the particle spectra, particularly below $1 \mathrm{MeV}$, leading up to the shock passage [8]. The fact that the SEP event was observed by several spacecraft positioned around the Sun has also provided the opportunity to examine SEP properties as a function of longitude, e.g., the proton and electron 
onsets and time profiles [6] and the heavy ion spectra and composition [7]. As solar activity continues to increase, it is expected that PSP will observe more events, hopefully closer to the Sun and in combination with other spacecraft.

\section{Acknowledgements}

This work was supported by NASA's Parker Solar Probe Mission, contract NNN06AA01C. Parker Solar Probe was designed, built, and is now operated by the Johns Hopkins Applied Physics Laboratory as part of NASA's Living with a Star (LWS) program. Support from the LWS management and technical team has played a critical role in the success of the Parker Solar Probe mission. We thank all the scientists and engineers who have worked hard to make PSP a successful mission.

\section{References}

[1] Fox, N. J., Velli, M. C., Bale, S. D., et al. 2016, SSRv, 204, 7

[2] Kasper, J. C., Abiad, R., Austin, G., et al. 2016, SSRv, 204, 131

[3] Bale, S. D., Goetz, K., Harvey, P. R., et al. 2016, SSRv, 204, 49

[4] McComas, D. J., Alexander, N., Angold, N., et al. 2016, SSRv, 204, 187

[5] McComas, D. J., Christian, E. R., Cohen, C. M. S., et al. 2019, Nature, 576, 223

[6] Kollhoff, A., Kouloumvakos, A., Lario, D., et al. 2021, A\&A, 10.1051/0004-6361/202140937

[7] Mason, G. M., Cohen, C. M. S., Ho, G. H., et al. 2021, A\&A, 10.1051/0004-6361/202141310

[8] Lario, D., Richardson, I. G., Palmerio, E., et al. 2021, ApJ, submitted

[9] Cohen, C. M. S., Christian, E. R., Cummings, A. C., et al. 2021, A\&A, submitted

[10] Pulupa, M., Bale, S. D., Bonnell, J. W., et al. 2017, JGR, 122, 2836

[11] Hill, M. E., Mitchell, D. G., Andrews, G. B., et al. 2017, JGR, 122, 1513

[12] Wiedenbeck, M. E., Angold, N. G., Birdwell, B., et al. 2017, PoS(ICRC2017)016

[13] Ellison, D. C. \& Ramaty, R. 1985, ApJ, 298, 400

[14] Cohen, C. M. S., Stone, E. C., Mewaldt, R. A., et al. 2005, JGR, 110, A09S16

[15] Tylka, A. J., Cohen, C. M. S., Dietrich, W. F., et al. 2005, ApJ, 625, 474

[16] Dresing, N., Theesen, S., Klassen, A., \& Heber, B. 2016, A\&A, 588, A17

[17] Blanco, J. J., Hidalgo, M. A., Gómez-Herrero, R., et al. 2013, A\&A, 556, A146

[18] Giacalone, J., Burgess, D., Bale, S. D., et al. 2021, A\&A, submitted

[19] Reames, D. V. 1999, SSRv, 90, 413 